\title{
Fermionic Superradiance in a Transversely Pumped Optical Cavity
}

\author{
J. Keeling, ${ }^{1}$ M. J. Bhaseen, ${ }^{2}$ and B. D. Simons ${ }^{3}$ \\ ${ }^{1}$ SUPA, School of Physics and Astronomy, University of St Andrews, St Andrews KY16 9SS, United Kingdom \\ ${ }^{2}$ Department of Physics, King's College London, Strand, London WC2R 2LS, United Kingdom \\ ${ }^{3}$ University of Cambridge, Cavendish Laboratory, Cambridge CB3 OHE, United Kingdom
}

(Received 10 September 2013; published 8 April 2014)

\begin{abstract}
Following the experimental realization of Dicke superradiance in Bose gases coupled to cavity light fields, we investigate the behavior of ultracold fermions in a transversely pumped cavity. We focus on the equilibrium phase diagram of spinless fermions coupled to a single cavity mode and establish a zero temperature transition to a superradiant state. In contrast to the bosonic case, Pauli blocking leads to lattice commensuration effects that influence self-organization in the cavity light field. This includes a sequence of discontinuous transitions with increasing atomic density and tricritical superradiance. We discuss the implications for experiment.
\end{abstract}

DOI: $10.1103 /$ PhysRevLett.112.143002

PACS numbers: $37.30 .+\mathrm{i}, 42.50 . \mathrm{Pq}$

Introduction.-Experiments combining cold atomic gases with cavity quantum electrodynamics have led to pivotal developments in matter-light interaction. The use of Bose-Einstein condensates (BECs) allows precise control over the collective matter-light coupling, and permits access to the strong coupling regime $[1,2]$. This may be exploited for spectroscopy of many body systems [3-5], and to induce light-mediated interactions.

Early theoretical work predicted that atoms in a cavity undergo self-organization when pumped transversely [6-8]. This was confirmed by experiments on thermal clouds and recently on a BEC [9-11]. The latter also established equivalence to the superradiance transition in an effective Dicke model [12-17]. The recent advances in optical cavities open the door to nonequilibrium and strongly correlated matter-light phenomena, including driven-dissipative phase transitions [18,19], Mott insulator transitions in self-organized lattices [20,21], cavity optomechanics [22], and bistability in ring cavities [23]. They also provide a platform on which to explore frustrated spin models and glassy behavior in multimode cavities [24-28]. For a review, see Ref. [29].

The experimental realization of superradiance in BECs raises many questions regarding the possible behavior of fermions in cavities. Recent investigations have considered longitudinal pumping [30,31], cavity mediated pairing [32], and synthetic gauge fields [33]. For earlier work on the complementary phenomenon of free-space superradiance and collective atomic recoil lasing using ultracold fermions, see Refs. [34-36]. In this Letter we focus on the closest extension of recent experiments $[10,11]$ by coupling spinless fermions to a single mode of a transversely pumped cavity. In contrast to dynamical superradiance in free space, steady-state superradiance may exist. At high temperatures, where both fermions and bosons exhibit Maxwell-Boltzmann statistics, it is evident that fermions will exhibit a superradiance transition, as observed for thermal bosons [9]. However, at low temperatures the Bose and Fermi gases are expected to behave differently and numerous questions arise. Does the self-organization transition survive for degenerate fermions? How does commensurability between the Fermi wave vector and the self-consistent optical lattice affect self-organization? Do new phases exist, and what characterizes the transitions?

Model.-Inspired by Refs. [9-11], we consider spinless fermions coupled to a single mode of a cavity light field (forming a standing wave in the $x$ direction), and pumped by a transverse laser (in the $z$ direction); see inset of Fig. 1 . We assume that the pump frequency $\omega_{p}$ is far detuned from the atomic transition frequency $\omega_{a}$ so that absorption and consequent heating via spontaneous emission can be neglected. After eliminating the excited states of the atoms (see, e.g., Ref. [37]), one obtains an effective Hamiltonian governing the interaction between the cavity light field and the motional degrees of freedom:

$$
\begin{aligned}
\hat{H}= & \hbar \omega \hat{\psi}^{\dagger} \hat{\psi}+\sum_{\mathbf{k}}\left[\frac{\hbar^{2} k^{2}}{2 m} \hat{c}_{\mathbf{k}}^{\dagger} \hat{c}_{\mathbf{k}}-\frac{g^{2}}{4 \Delta_{a}} \hat{\psi}^{\dagger} \hat{\psi} \sum_{s} \hat{c}_{\mathbf{k}}^{\dagger} \hat{c}_{\mathbf{k}+2 s \mathbf{q}_{x}}\right. \\
& \left.-\frac{g \Omega}{4 \Delta_{a}}\left(\hat{\psi}+\hat{\psi}^{\dagger}\right) \sum_{s, s^{\prime}} \hat{c}_{\mathbf{k}}^{\dagger} \hat{c}_{\mathbf{k}+s \mathbf{q}_{z}+s^{\prime} \mathbf{q}_{x}}-\frac{\Omega^{2}}{4 \Delta_{a}} \sum_{s} \hat{c}_{\mathbf{k}}^{\dagger} \hat{c}_{\mathbf{k}+2 s \mathbf{q}_{z}}\right]
\end{aligned}
$$

where $\Delta_{a} \equiv \omega_{a}-\omega_{p}$ is the difference between the atomic transition frequency $\omega_{a}$ and the pump frequency $\omega_{p}$, and $s$, $s^{\prime} \in \pm 1$ denote the forward and backward components of the standing waves. Here, $\hat{c}_{\mathbf{k}}$ is an annihilation operator for a spinless fermion with mass $m$ and wave vector $\mathbf{k}$, and $\hat{\psi}$ is a bosonic annihilation operator for cavity photons. Equation (1) is written in the rotating frame of the pump so $\omega \equiv \omega_{c}-\omega_{p}$ is the cavity-pump detuning. To ensure efficient scattering between the pump and the cavity, the 


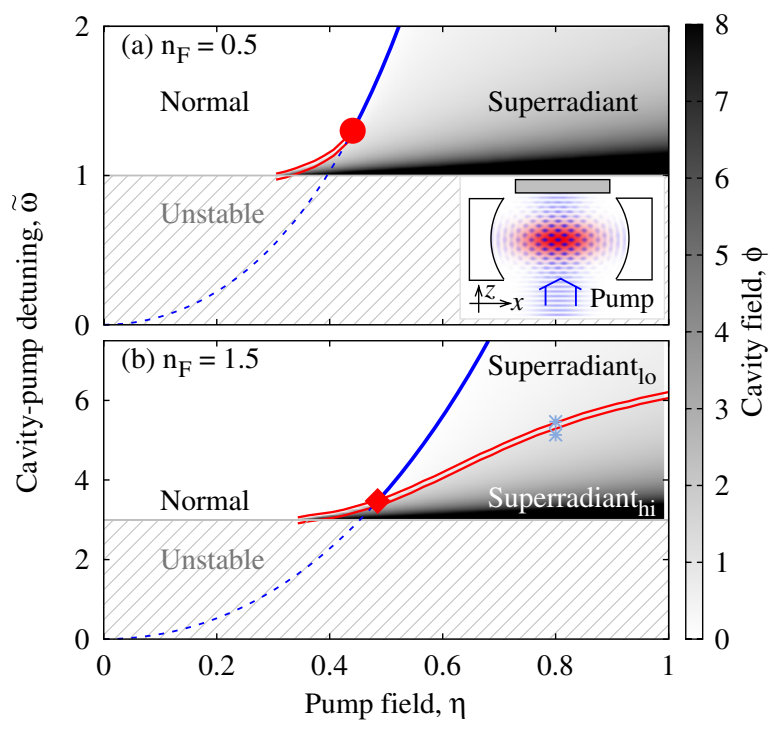

FIG. 1 (color online). Equilibrium phase diagram of fermions in a transversely pumped cavity; see inset. As the pumping is increased, there is a transition to a superradiant (SR) state, where the fermions spontaneously "self-organize" in the self-consistent light field. The panels correspond to partial filling of (a) the first and (b) second bands of the emergent lattice. At large detuning the cavity field (gray scale) grows continuously above a critical pump field (solid blue line), while at smaller detuning the transition is discontinuous (double red line). These first- and second-order boundaries join differently at different fillings; for $n_{F} \lesssim 1$ they meet at a tricritical point (circle), while at higher fillings there is a critical end point (diamond). The first-order boundary in (b) corresponds to a liquid-gas-type transition within the SR phase. The unstable region $\tilde{\omega}<2 n_{F}$ will be stabilized by cavity loss; see text. The dashed line is the spinodal extension of the continuous line.

cavity frequency $\omega_{c}$ is assumed to be close to $\omega_{p}$. The cavity and pump light fields thus have approximately equal wavelengths with $\left|\mathbf{q}_{x}\right|=\left|\mathbf{q}_{z}\right|=q$. Scattering between the pump and the cavity involves the cavity-atom coupling $g$, and the pump strength $\Omega$, and is described by the fourth term in Eq. (1). The third (fifth) term corresponds to a second-order process involving the absorption and emission of cavity (pump) photons. For bosons, there are limits where one may truncate the number of $\mathbf{k}$ states; when truncated to $\mathbf{k}=(0,0)$ and $\mathbf{k} \in( \pm q, \pm q)$, the Hamiltonian maps on to an effective Dicke model describing two-level systems coupled to light $[10,16,17,37]$. For fermions, Pauli blocking generally precludes truncation, and so we must consider the higher $\mathbf{k}$ states.

In a "self-organized" state, the cavity light field develops an expectation $\langle\hat{\psi}\rangle \neq 0$, and the superposition of the pump and cavity fields forms a $2 \mathrm{D}$ lattice with reciprocal lattice vector $(q, q)$. We introduce dimensionless units by measuring atomic energies in units of the recoil energy $E_{R} \equiv \hbar^{2} q^{2} / 2 m$. Wave vectors (and lengths) are measured in units of the magnitude of the reciprocal lattice vector $\sqrt{2} q$ so that the resulting Brillouin zone (BZ) has unit area.
We consider atoms confined to a 2D layer in the $(x, z)$ plane and thus define the filling fraction $n_{F} \equiv N / N_{l}$ as the number of atoms per lattice site; $N_{l}=2 q^{2} \mathcal{A} /(2 \pi)^{2}$ for a real space area $\mathcal{A}$. From Eq. (1) it is natural to introduce dimensionless pump and cavity fields via $\eta^{2}=\Omega^{2} / 4 \Delta_{a} E_{R}$ and $\phi^{2}=g^{2}\langle\hat{\psi}\rangle^{2} / 4 \Delta_{a} E_{R}$.

Equilibrium phase diagram.-We begin by determining the equilibrium phase diagram for the Hamiltonian (1). Although this neglects cavity losses, key features will survive in this limit $[10,11]$. We treat the cavity mode in mean-field theory, which is exact in the thermodynamic limit $N, \mathcal{A} \rightarrow \infty$ [38] . Considering the dimensionless freeenergy density $f=F /\left(E_{R} N_{l}\right)$, we find

$$
f=\tilde{\omega}|\phi|^{2}-\tilde{\beta}^{-1} \int_{\mathrm{BZ}} d^{2} k \sum_{i} \ln \left[1+e^{-\tilde{\beta}\left(\epsilon_{\mathbf{k}}^{(i)}-\mu\right)}\right]+\mu n_{F},
$$

where $\tilde{\omega} \equiv \omega\left(4 \Delta_{a} / g^{2} N_{l}\right)$ is a dimensionless cavity-pump detuning. Here, $\epsilon_{\mathbf{k}}^{(i)}$ is the energy in the $i$ th band, found by diagonalizing the atomic part of Eq. (1). Both $\epsilon_{\mathbf{k}}^{(i)}$ and $\mu$ are in units of $E_{R}$, and $\tilde{\beta} \equiv E_{R} / k_{B} T$. To ensure that $\mu$ is unambiguously defined, even for filled bands, we work at a low nonzero temperature, $k_{B} T=0.05 E_{R}$. Minimization of $f$ at fixed $n_{F}$ yields Fig. 1 .

Figure 1 shows two fillings, characteristic of a partially filled first band [Fig. 1(a), $n_{F}=0.5$ ] and a partially filled second band [Fig. 1(b), $n_{F}=1.5$ ]. In both cases, two phases exist. At low $\eta$ there is a normal state with $\phi=0$. At large $\eta, \phi \neq 0$, and this state is labeled "superradiant" (SR) by analogy with the Dicke model terminology [13-17]. It is also "self-organized" as the fermions are arranged in a self-consistent optical lattice.

Landau theory.-As found in the bosonic case [10,11], the normal-SR transition is second order at high $\eta$ and $\tilde{\omega}$. However, for the partially filled first band, on decreasing $\eta$, a tricritical point occurs beyond which the transition becomes first order. This can be understood via a Landau expansion, $f=f_{0}+a\left(\tilde{\omega}, \eta, n_{F}\right) \phi^{2}+b\left(\eta, n_{F}\right) \phi^{4}+$ $c\left(\eta, n_{F}\right) \phi^{6}$, where pump-cavity phase locking ensures $\phi \in \mathbb{R}$; see the Supplemental Material [39]. Taking $c>0$ for stability, three types of behavior occur depending on the value of $b$ [40]. For $b>0$, a continuous transition occurs at $a=0$, while a first-order transition occurs at $a=b^{2} / 4 c$ when $b<0$. These transitions meet at a tricritical point at $a=b=0$. In the vicinity of the tricritical point, the critical exponent describing the onset of the cavity field $\phi$ changes from $\beta=1 / 2$ to $\beta=1 / 4$. At low $\eta$, $b\left(\eta, n_{F}\right)<0$, and so the boundary becomes first order in Fig. 1(a).

The physical origin of this discontinuous transition is reminiscent of the Larkin-Pikin mechanism [41], where coupling to an additional degree of freedom drives a transition first order. Here, the order parameter $\phi$ couples to density waves of the atomic system. The linear coupling 
to the $\cos (x / \sqrt{2}) \cos (z / \sqrt{2})$ density wave described by the fourth term in Eq. (1) yields a continuous transition. However, the quadratic coupling to the $\cos (\sqrt{2} x)$ density wave in the third term in Eq. (1) drives $b<0$ at small $\eta$, as follows from second-order perturbation theory. As we discuss more fully in the Supplemental Material [39], such first-order transitions also occur in the full description of the bosonic problem, but are absent within the Dicke approximation. Note that this mechanism is distinct from the Brazovskii mechanism for driving the self-organization transition first order in multimode cavities $[24,25]$.

The behavior described thus far persists while only the first band is filled. Richer behavior occurs when higher bands start to be filled; see Fig. 1(b). Here, there is no tricritical point and the second-order line terminates at a critical end point [40]. The SR phase is now divided by a first-order transition, separating high and low $\phi$ regions. This is analogous to a liquid-gas transition, and the two phases are connected by a trajectory at lower $n_{F}$. Within Landau theory this corresponds to terms beyond $\phi^{4}$ being negative. We will return to the physical origin of this transition later in the Letter.

Unstable regions.-As indicated in Fig. 1, when $\tilde{\omega}<2 n_{F}, f$ is unbounded from below. This reflects the form of $f$ at large $\phi$, when the atoms are trapped in deep minima of the cavity optical lattice. Here, the leading contribution to the atomic energy is $\epsilon_{\mathrm{k}}^{(i)} \sim-2 \phi^{2}$, and so $f \sim\left(\tilde{\omega}-2 n_{F}\right) \phi^{2}$. The unstable region exists even at low densities, where Pauli blocking can be ignored, and so it is also relevant for bosons. Such a situation has been discussed for bosons in Ref. [42]. However, as we argued in Ref. [37], this instability will be replaced by dynamical attractors in the presence of cavity losses.

Continuous phase boundaries.-Where the boundaries are continuous, the $\eta, n_{F}$ dependence of the critical detuning $\tilde{\omega}$ can be obtained from the vanishing quadratic Landau coefficient. This has contributions from the first and third terms in Eq. (1). Using second-order perturbation theory, $a\left(\tilde{\omega}, \eta, n_{F}\right)=\tilde{\omega}+\chi\left(\eta, n_{F}\right)$, where

$$
\chi\left(\eta, n_{F}\right)=4 \eta^{2} \int_{\mathrm{BZ}} d^{2} k \sum_{i j} n\left(\epsilon_{\mathbf{k}}^{(i)}\right) \frac{\left|\left\langle u_{\mathbf{k}}^{(i)}\left|m_{1}\right| u_{\mathbf{k}}^{(j)}\right\rangle\right|^{2}}{\epsilon_{\mathbf{k}}^{(i)}-\epsilon_{\mathbf{k}}^{(j)}}
$$

is a dimensionless atomic susceptibility. Here, $\epsilon_{\mathbf{k}}^{(i)}$ and $\left|u_{\mathbf{k}}^{(i)}\right\rangle$ are the atomic energies and eigenstates evaluated in the absence of the cavity field and $n(\epsilon)$ is the Fermi-Dirac distribution. The pump-cavity scattering represented by the third term in Eq. (1) corresponds to $m_{1}=4 \cos (x / \sqrt{2}) \times$ $\cos (z / \sqrt{2})$ in the position basis. At $\phi=0$, the wave functions $\left\langle x, z \mid u_{\mathbf{k}}^{(i)}\right\rangle$ factorize into plane waves in the $x$ (cavity) direction and Mathieu functions [43] in the $z$ (pump) direction due to the pump lattice. The phase boundary occurs at $\tilde{\omega}=-\chi$; for parameters where the boundary turns first order, this becomes the spinodal line [40], as shown by the dashed lines in Fig. 1.
In the limits of low and high pump field, analytical results for the boundaries may be obtained. For $\eta \rightarrow 0$, the Mathieu functions are plane waves and one finds $\chi=-4 \pi \eta^{2}(1-\Theta(\gamma) \sqrt{|\gamma|})$, where $\Theta(\gamma)$ is a Heaviside step function and $\gamma=1-4 n_{F} / \pi$. The sharp threshold reflects Pauli blocking: at high densities the susceptibility saturates due to states deep within the Fermi surface (FS) not contributing. The threshold occurs when $n_{F}=\pi k_{F}^{2}=\pi / 4$ or $2 k_{F}=1$, i.e., when the FS diameter matches the scattering wave vector represented by the third term in Eq. (1). Note, however, that at low $\eta$ the phase boundary is actually first order, so $\tilde{\omega}=-\chi$ is a spinodal line. The $\eta^{2}$ dependence is evident from the dashed line in Fig. 1. In the low density and low pump field limit this corresponds to $\tilde{\omega}=8 \eta^{2} n_{F}$, which is where the Dicke approximation would erroneously predict a transition. As $\eta \rightarrow \infty$, the Mathieu functions are very localized so the dispersion in $k_{z}$ is flat, while remaining quadratic in $k_{x}$. The bands induced by the pump are well separated and so only the lowest band need be considered, yielding $\chi=16 \eta^{2} \ln \mid\left(1-n_{F}\right) /$ $\left(1+n_{F}\right) \mid$. The divergence at $n_{F}=1$ is a consequence of FS nesting. For a flat $z$ dispersion, the FS is delimited by $\left|k_{z}\right|<1 / \sqrt{2},\left|k_{x}\right|<n_{F} / 2 \sqrt{2}$, and the cavity-pump scattering induces atomic scattering, $k_{x} \rightarrow k_{x} \pm 1 / \sqrt{2}$, at a nesting wave vector for $n_{F}=1$. At finite $\eta$, the logarithmic singularity is softened by imperfect nesting, but a peak remains unless $\eta \ll 1$. We will return to this below.

Liquid-gas transition.-As noted earlier, for partial filling of the second (or higher) bands, a liquid-gas-type transition exists. The origin of this transition is revealed by the dispersion of the self-consistent bands with cavity field $\phi$, as shown in Fig. 2(a). The second band consists of $p_{x^{-}}$and $p_{z}$-like orbitals. Which of these orbitals has lower

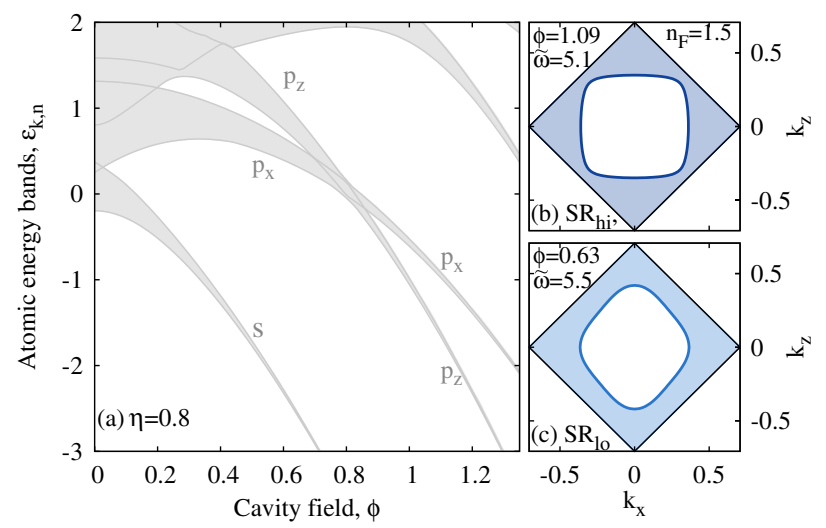

FIG. 2 (color online). (a) Atomic bands versus $\phi$ for $\eta=0.8$. When filling the second or higher bands, $f(\phi)$ is nonmonotonic, thus allowing first-order liquid-gas-type transitions in the SR phase. The bands are labeled by the symmetry of the Wannier orbitals at large $\phi$. Panels (b), (c) illustrate a distortion of the FS on crossing the liquid-gas boundary; the values of $\tilde{\omega}$ are indicated by crosses in Fig. 1(b). The FS delimits a partially filled second band and is shown in a reduced zone scheme. Occupied states are shaded. 
energy depends on the relative strength of $\phi$ and $\eta$ which control the lattice depth in the $x$ and $z$ directions, respectively. When $\phi \simeq \eta$, the $p_{x}$ - and $p_{z}$-like bands cross. This crossing leads to a kink in the atomic free energy at $\phi \simeq \eta$ and a maximum in $f(\phi)$ separating two minima. This yields a discontinuous jump from a high field state $\left(\mathrm{SR}_{\mathrm{hi}}\right)$ with $\phi \gtrsim \eta$ to a low-field state $\left(\mathrm{SR}_{\mathrm{lo}}\right)$ with $\phi \lesssim \eta$, accompanied by a distortion of the FS; see Figs. 2(b) and 2(c). More generally, the dispersion of the higher bands is nonmonotonic in $\phi$, and further band crossings can occur. Filling such bands leads to a nonmonotonic $f(\phi)$, and hence additional liquid-gas-type transitions.

Evolution with filling.-Further insight into the liquidgas transition is obtained from the phase diagram as a function of $n_{F}$ at fixed $\eta$; see Fig. 3(a). There is a critical point within the SR phase for $n_{F} \gtrsim 1$, beyond which a liquid-gas boundary extends. This corresponds to the point at which a sufficient fraction of the second band is filled in order to introduce the required local minima in $f(\phi)$. Also visible in Fig. 3(a) is a peak in the second-order boundary $\tilde{\omega}=-\chi$ near $n_{F}=1$. As $\eta$ increases, this evolves into the logarithmic singularity of $\chi$ at $n_{F}=1$, reflecting the FS nesting discussed above. As shown in Figs. 3(b) and 3(c), this peak survives over a range of temperatures $T \lesssim 0.2 E_{R}$, and with nonquadratic confinement. Combining such trapping potentials [44] with optical cavities is an area for experimental research.

Experimental considerations. - Thus far we have provided a detailed analysis of the equilibrium properties of Eq. (1). However, as discussed in Refs. [37,45], a finite

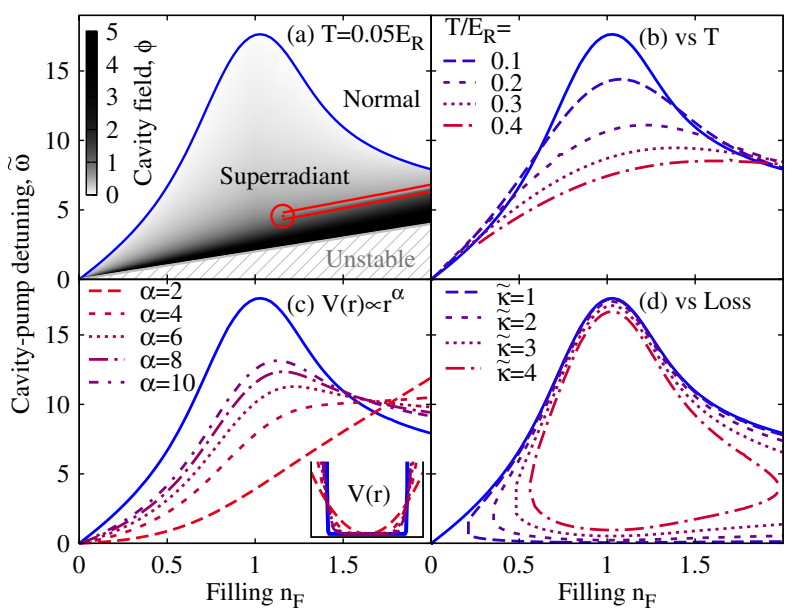

FIG. 3 (color online). (a) Equilibrium phase diagram as a function of $n_{F}$ at $\eta=0.8$. The lines and the gray scale are as in Fig. 1. A critical point (empty circle) terminates the liquid-gaslike transition at $n_{F} \gtrsim 1$. The second-order normal-SR transition shows a peak near $n_{F} \simeq 1$ reflecting nesting; see text. (b) Evolution with temperature. (c) Effect of a confining potential, $V(r)=E_{R}\left(r / r_{0}\right)^{\alpha}$, as shown in the inset; the solid line corresponds to a hard-wall potential. (d) Impact of cavity losses. In (b)-(d) we focus on the change to the (second-order) normal-SR boundary, and other features are suppressed. cavity decay rate $\kappa$ can significantly change the phase diagram. In a driven-open system, the phase diagram is found by determining the stable attractors of the dynamics, not by minimizing the free energy. Nonetheless, extrema of $f$ correspond to stationary points of the dynamics in the limit $\kappa \rightarrow 0$. The open system thus inherits key features from its equilibrium counterpart $[10,11]$. We next discuss which features are robust to nonzero $\kappa$, and which aspects require further investigation.

A feature that will survive when $\kappa \neq 0$ is the boundary at which the normal state becomes unstable. This can be calculated by finding when the normal mode frequencies have negative imaginary parts [46]. Following the approach used for the bosonic system [16,37], the boundary of stability is given by $\left(\tilde{\kappa}^{2}+\tilde{\omega}^{2}\right) / \tilde{\omega}=-\chi$, where $\chi$ is the atomic susceptibility given by Eq. (3) and $\tilde{\kappa} \equiv 4 \kappa \Delta_{a} / g^{2} N_{l}$; see Fig. 3(d). In the limit $\kappa \rightarrow 0$ one recovers the equilibrium result $\tilde{\omega}=-\chi$ discussed above. This provides a direct link between the equilibrium and nonequilibrium phase boundaries, as found for the open Dicke model [10,16,17]. As shown in Fig. 3(d), the commensuration peak survives over a range of $\tilde{\kappa}$ [47] .

Unfortunately, a quantitative discussion of the fate of the first-order boundaries is more challenging in the open fermionic system. Nonetheless, the existence of competing local minima in the equilibrium phase diagram suggests that multiple dynamical attractors may survive in the open limit. Likewise, determining the fate of the unstable region when $\kappa \neq 0$ is difficult because it hinges on the long-time dynamics of the fermionic system. This could potentially involve limit cycles, fixed points, and chaotic attractors [46]. Indeed, the answer to the analogous question in the Dicke model [37] demonstrates both superradiant phases and limit cycles. It would be interesting to explore this in more detail, both theoretically and experimentally.

Conclusions. - We have explored the phase diagram of ultracold fermions in a transversely pumped cavity. In contrast to a BEC, the interplay of the Fermi wave vector with the wavelength of the cavity field leads to a rich dependence on the filling fraction. We have established distinct superradiance transitions whose character reflects the impact of Pauli blocking and lattice commensuration. Unlike the Dicke model, the phase boundary turns first order at low pump field. In addition to signatures in the cavity light field, the measurable consequences include FS distortions and an enhanced susceptibility near unit filling. This study provides a basis for future experimental and theoretical research including the nature of dynamical attractors in the driven-dissipative system, the impact of fermion interactions, and the behavior in multimode geometries.

We thank T. Esslinger for discussions and B. L. Lev for helpful comments on the manuscript. M. J. B. and B. D. S. acknowledge EPSRC EP/J017639/1, and M.J.B. thanks the Thomas Young Centre. J. K. acknowledges support 
from EPSRC program “TOPNES" (EP/I031014/1), EPSRC (EP/G004714/2), and hospitality from KITP Santa Barbara. This research was supported in part by the National Science Foundation under Grant No. NSF PHY11-25915.

Note added.-Recently, two other works addressing a similar problem appeared $[48,49]$.

[1] F. Brennecke, T. Donner, S. Ritter, T. Bourdel, M. Köhl, and T. Esslinger, Nature (London) 450, 268 (2007).

[2] Y. Colombe, T. Steinmetz, G. Dubois, F. Linke, D. Hunger, and J. Reichel, Nature (London) 450, 272 (2007).

[3] I. B. Mekhov, C. Maschler, and H. Ritsch, Nat. Phys. 3, 319 (2007).

[4] W. Chen, D. Meiser, and P. Meystre, Phys. Rev. A 75, 023812 (2007).

[5] N. Brahms, T. P. Purdy, D. W. C. Brooks, T. Botter, and D. M. Stamper-Kurn, Nat. Phys. 7, 604 (2011).

[6] P. Domokos and H. Ritsch, Phys. Rev. Lett. 89, 253003 (2002).

[7] D. Nagy, J. K. Asbóth, P. Domokos, and H. Ritsch, Europhys. Lett. 74, 254 (2006).

[8] D. Nagy, G. Szirmai, and P. Domokos, Eur. Phys. J. D 48, 127 (2008).

[9] A. T. Black, H. W. Chan, and V. Vuletić, Phys. Rev. Lett. 91, 203001 (2003).

[10] K. Baumann, C. Guerlin, F. Brennecke, and T. Esslinger, Nature (London) 464, 1301 (2010).

[11] K. Baumann, R. Mottl, F. Brennecke, and T. Esslinger, Phys. Rev. Lett. 107, 140402 (2011).

[12] R. H. Dicke, Phys. Rev. 93, 99 (1954).

[13] K. Hepp and E. H. Lieb, Ann. Phys. (N.Y.) 76, 360 (1973).

[14] Y. K. Wang and F. T. Hioe, Phys. Rev. A 7, 831 (1973).

[15] C. Emary and T. Brandes, Phys. Rev. E 67, 066203 (2003).

[16] F. Dimer, B. Estienne, A. S. Parkins, and H. J. Carmichael, Phys. Rev. A 75, 013804 (2007).

[17] D. Nagy, G. Konya, G. Szirmai, and P. Domokos, Phys. Rev. Lett. 104, 130401 (2010).

[18] F. Brennecke, R. Mottl, K. Baumann, R. Landig, T. Donner, and T. Esslinger, Proc. Natl. Acad. Sci. U.S.A. 110, 11763 (2013).

[19] E. G. Dalla Torre, S. Diehl, M. D. Lukin, S. Sachdev, and P. Strack, Phys. Rev. A 87, 023831 (2013).

[20] J. Larson, B. Damski, G. Morigi, and M. Lewenstein, Phys. Rev. Lett. 100, 050401 (2008).

[21] J. Larson, S. Fernández-Vidal, G. Morigi, and M. Lewenstein, New J. Phys. 10, 045002 (2008).

[22] D. M. Stamper-Kurn, arXiv:1204.4351.

[23] D. Schmidt, H. Tomczyk, S. Slama, and C. Zimmermann, arXiv:1311.2156.

[24] S. Gopalakrishnan, B. L. Lev, and P. M. Goldbart, Nat. Phys. 5, 845 (2009).

[25] S. Gopalakrishnan, B. L. Lev, and P. M. Goldbart, Phys. Rev. A 82, 043612 (2010).
[26] S. Gopalakrishnan, B. L. Lev, and P. M. Goldbart, Phys. Rev. Lett. 107, 277201 (2011).

[27] P. Strack and S. Sachdev, Phys. Rev. Lett. 107, 277202 (2011).

[28] M. Müller, P. Strack, and S. Sachdev, Phys. Rev. A 86, 023604 (2012).

[29] H. Ritsch, P. Domokos, F. Brennecke, and T. Esslinger, Rev. Mod. Phys. 85, 553 (2013).

[30] J. Larson, G. Morigi, and M. Lewenstein, Phys. Rev. A 78, 023815 (2008).

[31] R. Kanamoto and P. Meystre, Phys. Rev. Lett. 104, 063601 (2010).

[32] X. Guo, Z. Ren, G. Guo, and J. Peng, Phys. Rev. A 86, 053605 (2012).

[33] B. Padhi and S. Ghosh, Phys. Rev. Lett. 111, 043603 (2013).

[34] M. G. Moore and P. Meystre, Phys. Rev. Lett. 86, 4199 (2001).

[35] W. Ketterle and S. Inouye, Phys. Rev. Lett. 86, 4203 (2001).

[36] P. Wang, L. Deng, E. W. Hagley, Z. Fu, S. Chai, and J. Zhang, Phys. Rev. Lett. 106, 210401 (2011).

[37] M. J. Bhaseen, J. Mayoh, B. D. Simons, and J. Keeling, Phys. Rev. A 85, 013817 (2012).

[38] The exactness of mean-field theory in the $N, \mathcal{A} \rightarrow \infty$ limit is discussed in the Supplemental Material [39]. This also occurs in the bosonic version of this model [50].

[39] See Supplemental Material at http://link.aps.org/ supplemental/10.1103/PhysRevLett.112.143002 for technical details of the calculation, including an extended discussion of first-order transitions and the susceptibility in a trap.

[40] P. M. Chaikin and T. C. Lubensky, Principles of Condensed Matter Physics (Cambridge University Press, Cambridge, England, 1995).

[41] A. I. Larkin and S. A. Pikin, Sov. Phys. JETP 29, 891 (1969).

[42] N. Liu, J. Lian, J. Ma, L. Xiao, G. Chen, J. Q. Liang, and S. Jia, Phys. Rev. A 83, 033601 (2011).

[43] E. T. Whittaker and G. N. Watson, A Course of Modern Analysis (Cambridge University Press, Cambridge, England, 1952).

[44] A. L. Gaunt, T. F. Schmidutz, I. Gotlibovych, R. P. Smith, and Z. Hadzibabic, Phys. Rev. Lett. 110, 200406 (2013).

[45] J. Keeling, M. J. Bhaseen, and B. D. Simons, Phys. Rev. Lett. 105, 043001 (2010).

[46] S. H. Strogatz, Nonlinear Dynamics and Chaos (Perseus Books, Cambridge, MA, 1994).

[47] In the bosonic case [10], $\kappa=8.1 \mathrm{MHz}$ and $g^{2} N / 4 \Delta_{a}=$ $13.3 \mathrm{MHz}$ for $N \sim 10^{5}$ bosons. This yields $\tilde{\kappa} \sim 0.6$.

[48] F. Piazza and P. Strack, following Letter, Phys. Rev. Lett. 112, 143003 (2014).

[49] Y. Chen, Z. Yu, and H. Zhai, this issue, Phys. Rev. Lett. 112, 143004 (2014).

[50] F. Piazza, P. Strack, and W. Zwerger, Ann. Phys. (Amsterdam) 339, 135 (2013). 